\title{
La guerra addosso. Conseguenze della Prima guerra mondiale sul corpo e sulla mente dei soldati
}

\author{
di Nina Quarenghi
}

Mag 24, $2021 \mid$ Didattica in classe, In evidenza $\mid \underline{0 \mid}$

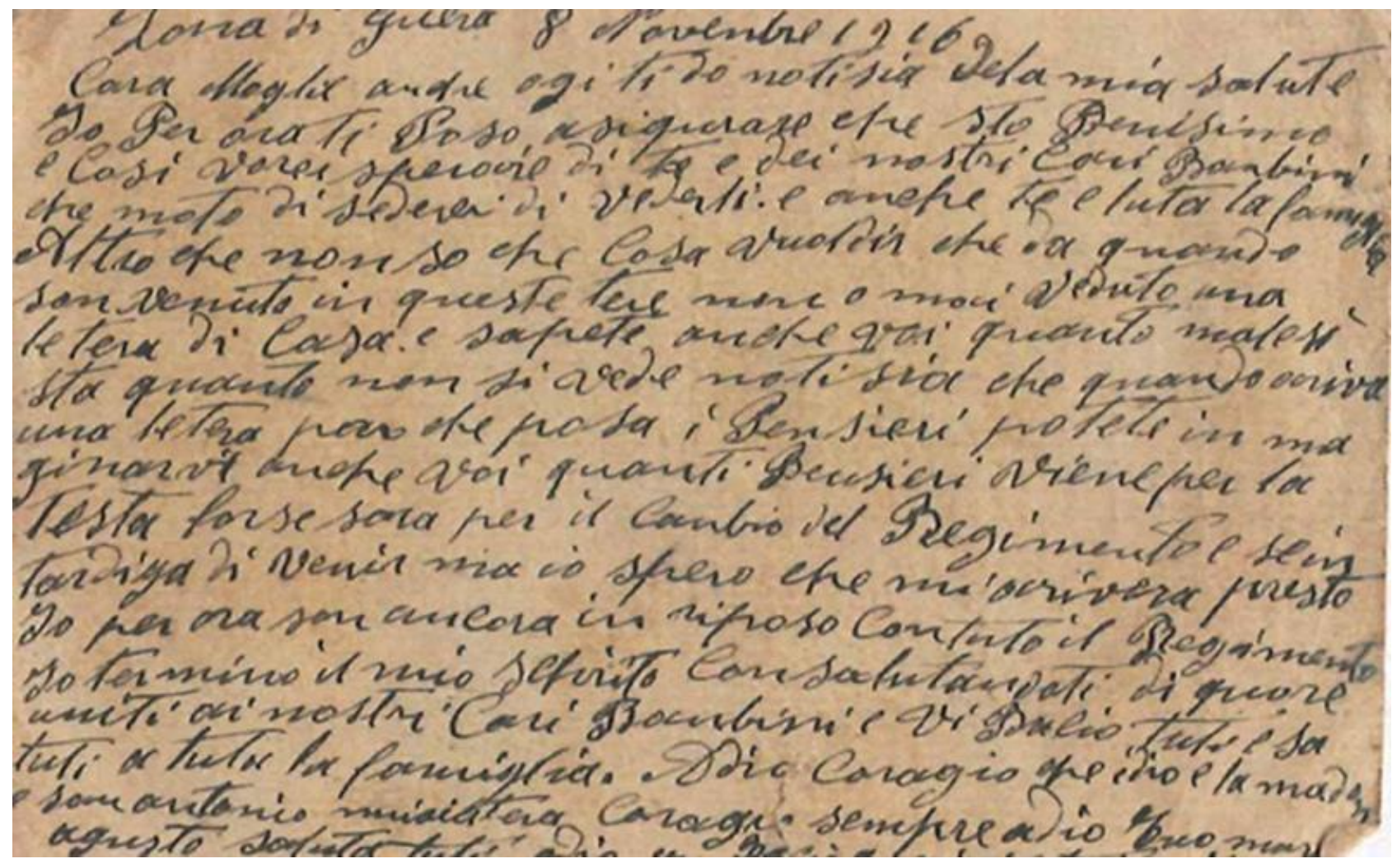

Foto tratta da https://www.irsifar.it/2020/11/29/1a-guerra-addosso/

\begin{abstract}
Durante la Grande Guerra la modernità irruppe nel panorama bellico, con la conseguenza che milioni di combattenti, al di là della divisa indossata, portarono per sempre impressi nel corpo e nello spirito i devastanti effetti prodotti dai nuovi armamenti. Fu un conflitto inaspettatamente lungo, nel quale si dispiegò il potenziale accumulato dalle tecnologie produttive e distruttive di tutti i Paesi coinvolti, costringendo milioni di individui a convivere quotidianamente con l'orrore della trincea. Lo scempio dei corpi dei soldati, dopo un attacco, si accompagnò ai danni psichici di molti di loro: furono decine di migliaia gli uomini, appartenenti a tutti gli schieramenti, che pur rimanendo integri nel corpo dopo cannoneggiamenti e assalti, rimasero menomati nella mente; lo studio della loro drammatica sorte è utile per comprendere come la pazzia possa essere considerata un irrazionale tentativo di fuga da quella che in effetti rappresentò la vera follia: la guerra di massa.
\end{abstract}


L'attività didattica qui proposta nasce dalla rielaborazione dei contenuti della mostra La guerra addosso. Tracce del primo conflitto mondiale sui corpi e nelle menti dei soldati, realizzata da Ludovico Testa nel 2018, e dalla relazione di Antonio Gibelli, I traumi nervosi e mentali dei soldati al fronte, all'interno del corso di formazione dal titolo La vittoria mutilata organizzato presso l'Irsifar di Roma nell'autunno del 2019.

È rivolta alle ultime classi delle scuole secondarie di primo e secondo grado e ha la durata minima di due ore, estendibili a tre-quattro se agli studenti viene chiesto anche il recupero delle informazioni e una riflessione finale sul senso complessivo dell'attività.

- 45 minuti: spiegazione dell' argomento attraverso un PowerPoint (SCARICA QUI) La spiegazione può essere sostituita dalla visione del documentario Scemi di guerra. La follia nelle trincee, di Enrico Verra, 2008, che si può trovare a questo link: https://www.raiplay.it/video/2018/04/La-follia-nelle-trincee-6baf6b4b-393c-43e8-8ec313430e60086f.html)

- Attività sui documenti (SCARICA QUI)

- 30 minuti: la classe viene divisa in gruppi di 3-4 studenti, ciascuno dei quali deve analizzare un documento, servendosi di una scheda-guida (SCARICA QUI)

- 30 minuti: i gruppi a turno relazionano alla classe i risultati della loro analisi.

Per gli insegnanti, sono disponibili anche le trascrizioni dei testi più complessi ed eventuali informazioni di contesto, che possono essere utili durante il momento finale dell'attività (SCARICA QUI).

\section{Documenti}

Alcuni documenti proposti per l'attività didattica provengono dal progetto La Grande Guerra, $i$ diari raccontano, nato dalla collaborazione dell'Archivio Diaristico Nazionale e il Gruppo L'Espresso; altri sono stati forniti dallo storico Antonio Gibelli e provengono dagli archivi della Provincia di Genova e dell'Ospedale Psichiatrico di Cogoleto; questi documenti, inediti, e fanno parte di un grande lavoro di ricerca, condotto dallo storico su questi temi, confluito nelle sue pubblicazioni, in particolare in L'officina della guerra. La Grande Guerra e le trasformazioni del mondo mentale, Bollati Boringhieri, Torino, 2007. Le immagini sono state prese dal catalogo della mostra La guerra addosso, curato da Ludovico Testa e Costantino Di Sante.

Queste fonti si possono scaricare in due versioni: una destinata agli studenti, senza nessuna didascalia, perché possano analizzare copie dei documenti originali; l'altra per i docenti, con le trascrizioni dei testi più complessi ed eventuali informazioni di contesto, che possono essere utili durante il momento finale dell'attività, quando gli studenti riferiscono il risultato del loro lavoro di analisi.

\section{Una guerra totale}

Per i milioni di uomini che vi presero parte[1] la Grande Guerra fu un'esperienza totalizzante: la macchina bellica imponeva al soldato disciplina e obbedienza, inserendolo in un ingranaggio nel quale l'autonomia del singolo veniva annullata. Di giorno e di notte, in ogni stagione, questi uomini vissero ininterrottamente in pochi metri quadrati di terra, roccia o ghiaccio, dove la tensione per un attacco subìto o un assalto comandato si alternava al logoramento di ore interminabili vissute in condizioni disumane. 
Questo accadde perché per la prima volta, al servizio della guerra, furono impiegate in modo massiccio le invenzioni tecnologiche della seconda rivoluzione industriale: elettricità, acciaio e chimica, portarono alla creazione in grande quantità di armi micidiali, che avrebbero dovuto risolvere un conflitto mondiale velocemente, mentre ebbero l'effetto contrario: il meccanismo automatico della morte sembrava non poter finire mai; la conseguenza fu un massacro di vite senza precedenti.

\section{Le guerre prima della Grande guerra}

Per comprendere come la Prima guerra mondiale rappresenti qualcosa di tragicamente nuovo rispetto al passato e sveli i paradossi della modernità, è utile confrontarla con i conflitti che la precedettero di pochi decenni. Le campagne belliche ottocentesche non erano guerre totali: le forze armate erano impegnate, solitamente nei mesi caldi, in una serie di battaglie, che si svolgevano nell'arco di una giornata e si concludevano quando una delle due parti riusciva a mettere in fuga l'altra; al sopraggiungere del buio i combattenti si ritiravano negli accampamenti, in attesa di nuove marce e battaglie. Queste ultime avvenivano perlopiù in spazi aperti, dove gli eserciti, distanti tra loro alcune centinaia di metri, cominciavano a colpirsi con le rispettive artiglierie e si avvicinavano progressivamente, fino a che non entravano in azione i fucili ad avancarica dei fanti, mentre la cavalleria, con le sue incursioni, cercava di sfondare lo schieramento avversario. In realtà la parte decisiva del combattimento era quello finale ravvicinato, all'arma bianca, quando i soldati e i loro ufficiali si buttavano nella mischia del corpo a corpo con baionette, sciabole e spade.

Nelle guerre dell'Ottocento i militari indossavano divise dai colori accesi, per non perdere il riferimento del proprio reggimento nella confusione dello scontro, tra la polvere sollevata da uomini, cannoni e cavalli e il fumo dell'artiglieria. Gli squilli delle trombe e il rullo dei tamburi erano necessari per guidare i combattenti nelle manovre e si univano al frastuono dei cannoni, al clangore delle spade e alle urla degli uomini in un crescendo di eccitazione e ferocia, che comunque si concludeva nel giro di alcune ore. Insomma, il "gioco" mortale aveva un inizio e una fine, sia in termini di battaglia che di campagna di guerra. Tutto ciò non si verificò nella Prima guerra mondiale, dove i tentativi di assaltare la linea nemica, nel giro di pochi minuti, lasciavano sul campo migliaia di uomini, senza che il fronte si spostasse di un metro in un senso o nell'altro. E questo durò ininterrottamente per quasi cinque anni.

\section{La prima guerra del Novecento}

Le guerre combattute tra la fine del XIX e l'inizio del XX secolo anticiparono la tragedia del primo conflitto mondiale, ma su scala inferiore: nella guerra anglo-boera, combattuta in Sudafrica tra britannici ed ex coloni olandesi, vennero impiegate le prime mitragliatrici automatiche; durante la guerra russo-giapponese (1904-05), in Manciuria e Corea, che cominciò ad avere le caratteristiche di una guerra di massa dominata dall' artiglieria, i medici curarono ferite devastanti e rilevarono i primi casi di nevrosi, che insorgeva nei combattenti dopo le battaglie, come dopo un cataclisma; nella guerra italo-turca (1911-12) per la conquista della Libia, i progressi tecnologici portarono all'utilizzo dei primi mezzi a motore, come automobili, motociclette e aeroplani, questi ultimi usati più per la ricognizione che per l'offesa. La mobilitazione di uomini e mezzi in questi conflitti fu tuttavia circoscritta ad alcuni Paesi, contrariamente alla Prima guerra mondiale, epopea che, per le sue dimensioni, colpì e angosciò buona parte della popolazione mondiale. 
A differenza del soldato dell'Ottocento, che era scosso da forti emozioni solo nelle giornate campali, quello in trincea viveva in un costante stato di allarme, tanto che E. J. Leed, nel suo Terra di nessuno, ha definito quella del 1914-18 una "guerra di nervi".[2] Un ufficiale medico italiano, nel suo libro di memorie, ben descrive la condizione dei soldati:

Vita sotterranea, umido, freddo, disagi alimentari, patimenti dello spirito provocati dalla estenuante asperità del soggiorno, così lungi dalle tenerezze familiari e tormentato dalla infernale paurosità degli strumenti guerreschi. E i gas asfissianti. [...] Ferite estese, da sgomentare il chirurgo ancor più del ferito.[3]

Come si arrivò a tutto questo? Per quale motivo gli alti comandi di tutti gli eserciti decisero di far vivere costantemente i loro uomini in un reticolo di fossati scavati nella terra o nella roccia?

Era accaduto che le nuove armi tecnologiche impedivano ai soldati di arrivare al corpo a corpo, perché venivano falciati prima di raggiungere la trincea nemica. La guerra si trasformò, da una sequenza di battaglie campali diluite nel tempo, in una macchina in cui la carne stessa dei soldati, dopo essersi macerata nell'attesa, veniva tritata dagli obici, dalle schegge di granata e dalla mitragliatrice. L'unico modo per non perdere la posizione, riparandosi da questo inferno, era scavare la trincea e viverci.

I soldati divennero un tutt'uno con il terreno; le loro divise non avevano più i colori sgargianti degli eserciti dell'Ottocento, ma le tonalità di grigio, verde, marrone e nel momento in cui le condizioni meteorologiche li ricoprivano di fango, la mimetizzazione era perfetta. Gli eserciti si distinguevano dalla forma degli elmetti, che comparvero sulle teste dei soldati, per quanto inefficaci davanti ai proiettili e alle schegge di granata. Cambiò anche il modo di combattere degli ufficiali, che non potevano più restare esposti in luoghi elevati a gestire le operazioni belliche, ma spesso manovravano gli assalti da un punto riparato della trincea, calcolando quanti uomini poteva fermare la mitragliatrice nemica per mandarne qualcuno in più, in un susseguirsi di assalti inconcludenti.

Nel giro di alcuni mesi, la parola sulla bocca di tutti, sia di chi si trovava a combattere in alta quota, che tra le ondulate pianure del fronte occidentale e orientale, era "immobilità".

\section{L'uomo e la natura della Grande guerra}

Anche la distruzione della natura fu causa di forte turbamento per i militari. Il paesaggio venne sacrificato e scarnificato, piegato alla volontà distruttrice della guerra tecnologica e restò mutilato al pari degli uomini e degli animali.

Gli alberi giacciono abbattuti, oppure sono scomparsi, fatti a pezzi, e ne resta solo qualche radice estirpata. I margini della strada sono sollevati e devastati dalle granate. E per tutta la sua lunghezza, quella strada dove sono rimaste in piedi solo le croci, è affiancata da trincee venti volte crollate e riaperte [...]. Più si va avanti e più tutto è devastato, putrefatto, terremotato. [4]

Questo scenario apocalittico colpì tutti i soldati, dai contadini analfabeti, avvezzi al silenzio e all'ordine della vita dei campi, ai cittadini istruiti, che restarono egualmente scossi per queste visioni contro natura: 
Una generazione che era andata a scuola col tram a cavalli, si trovava, sotto il cielo aperto, in un paesaggio in cui nulla era rimasto immutato tranne le nuvole, e sotto di esse, in un campo magnetico di correnti ed esplosioni micidiali, il minuto e fragile corpo dell'uomo.[5]

La Grande Guerra fu la traduzione e l'espressione in chiave militare della massificazione della società che era allora in atto. Di fronte a cannoni invisibili, che colpivano da lontanissimo senza preavviso con il frastuono di mille temporali, davanti al gas asfissiante, ai riflettori che rendevano possibili gli attacchi anche di notte, sovvertendo il ritmo della veglia e del sonno, il soldato restava quello che era: una piccola creatura fatta di carne, ossa, nervi e anima. E in questa gigantesca macchina, dove costituiva un «bullone che gira gira e poi viene buttato via»[6], l'uomo restò intrappolato, come in un labirinto[7], la cui uscita primaria era la corsa verso la morte.

\section{Tentativi di fuga}

Evadere da questo labirinto era impossibile, ma gli uomini riuscirono a farlo percorrendo alcune strade secondarie. Una di queste era la scrittura.[8]

Negli anni della Grande Guerra vennero scritti migliaia di diari personali e scambiate, tra i soldati al fronte e i loro cari a casa, circa quattro miliardi di lettere e cartoline[9]; un fiume di carta che sostenne i combattenti e consentì loro di astrarsi dalla disumanità che li circondava per proiettarsi nella normale esistenza degli affetti; la scrittura permise loro di pensare alla vita passata e sperare in quella futura, uscendo da un presente di freddo, di fame e di morte.

Altre vie di evasione, più drammatiche per le conseguenze che comportarono, furono la diserzione e l'autolesionismo. La scelta di tentare una fuga vera e propria riguardò un'esigua parte dei combattenti, per la difficoltà di attuare questa soluzione. Due erano gli impedimenti che scoraggiavano il soldato dal pianificare una diserzione: il primo era la propria coscienza, il sentimento di vergogna conseguente a un atto che era considerato da tutti l'emblema stesso della vigliaccheria; il secondo ostacolo veniva dalle strategie drastiche messe in atto dagli alti comandi per il contenimento delle fughe: punizioni esemplari, rappresaglia e fucilazione di commilitoni del disertore, in rapporto di dieci a uno.[10]

Altri soldati tentarono di lasciare il fronte ferendosi da soli, simulando un incidente. Difficilmente questa strategia diede l'esito sperato: procurarsi una lesione non grave, ma tale da poter essere allontanati in modo definitivo dalla guerra, non era facile; spesso chi scelse questa strada si ferì seriamente provocandosi danni irreparabili, oppure, restando ferito in modo lieve, venne facilmente curato e rispedito al fronte in tempi relativamente brevi.

Chi non riuscì a elaborare un piano razionalmente, ma non tollerò oltre la permanenza nella trappola della guerra, chi insomma fu dilaniato dal conflitto interiore tra il desiderio di sopravvivere e la sofferenza che la disciplina gli provocava, elaborò inconsciamente una nuova e più dolorosa via di fuga: la nevrosi o la pazzia. Il dolore venne somatizzato in infermità mentale, la sofferenza in un blocco di memoria. Era una difesa del corpo, una ribellione estrema del sistema nervoso, dei sensi e dello spirito.

Come vedremo nei prossimi paragrafi, mentre il ferito nel corpo, nonostante il disastroso scempio del suo fisico, venne a guerra conclusa acclamato come eroe e fu, anche se con fatica, reinserito nella società, chi rimase segnato nella mente subì un trattamento peggiore, a partire dal nome con il quale venne chiamato per il resto della vita: "scemo di guerra". 


\section{La guerra addosso: i feriti nel corpo}

Della distruzione fisica dei soldati della Prima guerra mondiale, troviamo testimonianza nelle relazioni degli ufficiali medici, che mai si erano trovati a dovere affrontare un simile massacro:

Appena tagliate con un colpo di forbici le fasciature frettolose - scrive un medico franceseappaiono le ferite, le ferite raccapriccianti degli obici, delle torpedini e delle granate, che scavano nelle carni fosse e mine, strappando muscoli, stritolando ossa, recidendo i nervi e i tendini. Tutto è chiazzato, tutto sanguina e geme. Schiacciamenti, spappolamenti di carne. Di questa mano non resta più che un mignolo appeso al lembo; di questo viso non ci son più che rimasugli di naso e di bocca; di questa coscia, nient'altro che un ammasso sanguinante di ossa e muscoli. Polmoni perforati, cervelli schizzati fuori, vesciche lacerate, intestini scoperti: qui ci sono brandelli di tutto il corpo umano.[11]

Questo scenario terrificante si presentava normalmente agli occhi dei medici di tutti gli eserciti, che si occupavano dei feriti negli ospedali da campo, dopo che, nelle ambulanze precarie prossime al fronte, erano state prestate le prime cure.

I metodi chirurgici spartani, utilizzati precipitosamente nel primo soccorso mentre ancora infuriava l'attacco, talvolta erano criticati da chi operava nei presidi medici successivi, soprattutto se erano state amputate malamente, sull'onda dell'urgenza, le parti del corpo ferite, con la conseguenza che il soldato moriva dissanguato. Viceversa c'era chi caldeggiava l'amputazione per impedire l'insorgere di infezioni. In un modo o nell'altro intervenire dopo quelle carneficine, oltre tutto con i poveri mezzi di allora, era impresa tanto difficile quanto vana e le guarigioni avevano del miracoloso, a meno che non si trattasse di ferite di piccola entità, sebbene anche in quel caso non si potesse stare tranquilli:

Ferite piccole e maliziose. Quasi invisibile la porta d'entrata di una scheggia entro il corpo del povero ferito; recisa in profondità la midolla spinale, un cordoncino esile come un dito, labile come gelatina, in cui è tuttavia compendiata ogni possibilità di sentire e di muoversi di mezzo organismo.[12]

Per i feriti che rimasero menomati per sempre nacque nel 1917 in Italia l'Associazione nazionale fra mutilati e invalidi di guerra (Anmig), che si mobilitò per la cura dei soldati e il sostegno alle loro famiglie. I reparti ortopedici crebbero nello sforzo di "ricostruire" i corpi e la creazione delle protesi permise agli uomini il ritorno a una vita pressoché normale, anche se colma di difficoltà.

I corpi martoriati di questi soldati, tuttavia, anziché rappresentare il volto osceno della guerra, che sfigura l'uomo e ne contraffà i segni stessi della sua identità, vennero al contrario sublimati e sacralizzati, quando, negli anni Venti e Trenta, si rafforzò l'operazione nazionalista di monumentalizzare la Grande Guerra da parte di tutti i paesi belligeranti, in particolare dell'Italia fascista. Il Milite ignoto, che diede la vita per la Patria, insieme al Grande mutilato, che sull'altare sacrificò un pezzo di corpo, furono esaltati come le figure mitiche dell'eroismo patrio.

Da quest'operazione retorica, che comunque riabilitava i mutilati e li restituiva alla società, furono esclusi i folli di guerra, che rimasero derisi ai margini dei villaggi o sparirono del tutto, inghiottiti per sempre dai manicomi. 


\section{La guerra addosso: $i$ feriti nella mente}

La distruzione mentale dei soldati della Prima guerra mondiale è testimoniata dai medici degli ospedali psichiatrici:

Talora questi malati sono confusi, attoniti, smarriti: più spesso sono ottusi, torpidi, inerti, con rallentamento delle funzioni mentali, depressi: somaticamente denutriti, pallidi con lo sguardo spento, con espressione stanca, stordita preoccupata, triste. [...] Presentano persistenza di immagini belliche a carattere ossessivo, ravvivanti stati emotivi penosi: hanno sonni agitati, con sogni paurosi, a contenuto bellico.[13]

Attraverso queste parole entriamo in contatto con la faccia della medaglia più tragica della guerra di massa: la storia di quei soldati che, travolti dall'orrore, reagirono perdendo il contatto con una realtà insopportabile, nella quale tuttavia rimasero intrappolati per sempre.

Il primo dramma per queste persone fu il mancato riconoscimento, da parte dei superiori e dei sanitari stessi, della loro condizione di feriti: non presentando nessuna lesione evidente, venivano considerati semplicemente dei deboli, dei vili. C'era uno sguardo di disprezzo, di riprovazione e di sfiducia su di loro, molto lontano dalla pietà che suscitava il ferito nel corpo.

Quando, tuttavia, era chiaro che questi soldati non potevano più prendere parte alla guerra, venivano internati nei manicomi e si cominciava un trattamento, che avrebbe dovuto ricondurli il prima possibile al fronte, essendo sani e forti nel fisico, quindi capaci perfettamente di tornare a fare numero.

Durante una prima fase di osservazione, gli psichiatri cercavano di distinguere i simulatori, che venivano immediatamente rispediti al fronte, da coloro che soffrivano effettivamente di un disagio psichico. Ci si occupava dunque di questi ultimi, cercando di renderli di nuovo abili alla guerra, senza riconoscere che l'origine del loro processo morboso fosse proprio la vita militare. La guerra era cosa sacra, che fortificava l'uomo anziché annientarlo; era dunque inconcepibile che fosse causa di malattia. In altre parole si riteneva che questi individui fossero già matti prima di diventare soldati, che fossero già deboli e compromessi e che un trattamento scioccante, doloroso, punitivo, fosse necessario per far loro recuperare quel minimo di lucidità che li avrebbe restituiti alla vita militare. Vennero per esempio utilizzate su di loro le scariche elettriche, ultimo ritrovato in campo psichiatrico, secondo il concetto che se i ricoverati avessero provato uno spavento più forte della guerra, sarebbero guariti; questi uomini fecero da cavia ai primi elettroshock della storia, tortura inutile che peggiorò ulteriormente il loro stato.

Per coloro che rimasero traumatizzati per sempre non vi fu riconoscimento da parte della società e dello Stato al termine della guerra; nessuna medaglia appuntata sul petto, nessuna protesi a compensare la loro mutilazione interiore, nessuna voce autorevole, durante le feste della patria, a ricordare il loro sacrificio. La loro miseria non poté essere sacralizzata, perché non era funzionale al mito della Nazione.

Le ricerche approfondite su questi sfortunati soldati, a partire dagli anni Settanta, da parte di storici del calibro di Antonio Gibelli, e le mostre come La guerra addosso di Ludovico Testa, hanno restituito loro la dignità di uomini, mettendo in luce non tanto la loro mente malata, ma la follia di chi progettò e volle la Prima guerra mondiale. 
- G. Boschi, La guerra e le arti sanitarie, Mondadori, Milano, 1935

- C. Di Sante, L. Testa, (a cura di), catalogo della mostra La guerra addosso. Tracce del primo conflitto mondiale sui corpi e nelle menti dei sopravvissuti, 2019

- E. Forcella, A. Monticone, Plotone di esecuzione. I processi della Prima guerra mondiale, Laterza, Bari, 2014

- A. Gibelli, L'officina della guerra. La Grande Guerra e le trasformazioni del mondo mentale, Bollati Boringhieri, Torino, 2007

- $\quad$ E. J. Leed, Terra di nessuno. Esperienza bellica e identità personale nella prima guerra mondiale, Il Mulino, Bologna, 1985

- S. Linden, La guerra dei nervi. Soldati traumatizzati sul fronte occidentale-1914/1918, Guida, 2021

Note:

[1] Le dimensioni della catastrofe: 67,4 milioni gli uomini mobilitati durante il conflitto, 10 milioni i militari morti in combattimento, 6,5 milioni i militari invalidi e mutilati, di cui 300.000 sfigurati da ferite al volto, 650.000 militari colpiti da "nevrosi bellica", 1.778 soldati condannati alla fucilazione, come esempio per gli altri. I dati sono contenuti nel catalogo della mostra La guerra addosso. Tracce del primo conflitto mondiale sui corpi e nelle menti dei sopravvissuti, a cura di Ludovico Testa e Costantino Di Sante.

[2] E. J. Leed, Terra di nessuno. Esperienza bellica e identità personale nella prima guerra mondiale, Il Mulino, Bologna, 1985. Il libro analizza le trasformazioni psicologiche e caratteriali subite dai partecipanti al conflitto, che divennero estranei perfino a loro stessi.

[3] G. Boschi, La guerra e le arti sanitarie, Mondadori, Milano, 1935, in A. Gibelli, L'officina della guerra. La Grande Guerra e le trasformazioni del mondo mentale, Bollati Boringhieri, Torino, 2007, p. 66. Le pagine suggestive di questo libro sono fondamentali per comprendere i cambiamenti provocati dalla guerra sulla percezione della realtà e a definire la Prima guerra mondiale come spartiacque decisivo nella storia contemporanea.

[4] H. Barbusse, il Fuoco, romanzo autobiografico pubblicato nel 1916, in cui l'autore narra la sua esperienza di soldato dell'esercito francese sul fronte occidentale.

[5] W. Benjamin, Il narratore. Considerazioni sull'opera di Nicola Leskov, in Angelus novus, Einaudi, Torino, 1962, p. 258.

[6] La storica Bruna Bianchi riporta nel documentario Scemi di guerra, la voce di uno dei soldati coinvolti nel conflitto, che descrive così la sua situazione di combattente.

[7] La descrizione della guerra come un labirinto è da attribuire a E. J. Leed, cfr. Un'uscita dal labirinto, in Terra di nessuno, cit. 
[8] Sul senso della scrittura in trincea cfr. l'articolo di F. Croci in Novecento.org:

http://www.novecento.org/pensare-la-didattica/parole-in-trincea-la-memoria-della-grande-guerranelle-testimonianze-scritte-dei-soldati-1868/

[9] Molto utile a questo proposito il progetto La Grande Guerra, i diari raccontano, nato dalla collaborazione dell'Archivio Diaristico Nazionale di Pieve Santo Stefano e il Gruppo L'Espresso, grazie al quale sono state selezionate e suddivise per temi centinaia di pagine di lettere di soldati e dei loro familiari: http://archiviodiari.org/index.php/iniziative-e-progetti/la-grande-guerra.html

[10] Cfr. E. Forcella, A. Monticone, Plotone di esecuzione. I processi della Prima guerra mondiale, Laterza, Bari, 2014. Pubblicato per la prima volta nel 1968, questo libro raccoglie i documenti e le sentenze di condanna emesse contro i soldati giudicati "disfattisti" o ribelli e fa luce sull'attività repressiva dei tribunali militari durante la guerra.

[11] Relazione di un ufficiale medico, contenuta in G. Boschi, La guerra e le arti sanitarie, pp. 8687

[12] G. Boschi, La guerra e le arti sanitarie, cit., in A. Gibelli, L'officina della guerra. La Grande Guerra e le trasformazioni de mondo mentale, cit. p. 66.

[13] G. Pellacani, Le neuropatie emotive e le psiconevrosi dei combattenti, «Rivista Sperimentale di Freniatria», XLIII (1919), in A. Gibelli, L'officina della guerra, cit. p. 127. 